\title{
Study on the Development and Utilization of Renewable Energy in China
}

\author{
Guizhou Ren ${ }^{1}$,Fangqing Li ${ }^{1}$,Gang Liu ${ }^{1,2}$, JianXin Ren ${ }^{1}$,Yudong Liu ${ }^{1}$ and Honghong Shen ${ }^{1}$ \\ ${ }^{1}$ School of Mechanical and Energy Engineering, Shanghai University of Electric Power, China \\ ${ }^{2}$ Guodian Tongling Power Generation,Co.Ltd
}

\begin{abstract}
With the continuous consumption of fossil energy and non-renewable, countries all over the world pay more and more attention to the use of renewable energy, among which Denmark, Japan, Germany and so on are among the best. Based on the basic national conditions and the energy development situation of our country, the utilization of renewable energy in our country is also promoted to the national strategic position. Experience shows that large-scale renewable energy access to the power grid system can not only meet the electricity demand of industrialized countries. Moreover, the safe and stable operation of the power grid can be realized by advanced technical means. Through the analysis of some advanced technology and equipment research in the development and utilization of renewable energy in foreign power grid, in order to improve the repeatability of our country, The level of development and utilization of raw energy.
\end{abstract}

\section{Introduction}

Energy is the basis of human life and industrial activities, but it is also the main source of greenhouse gases. Energy consumption produces about $60 \%$ of global greenhouse gas emissions. Due to excessive dependence on fossil energy, greenhouse gas emissions continue to increase, which brings serious challenges to human beings. Since the oil crisis of the 1970s, countries have realized the importance of the energy problem, and the investment trend of new energy has risen steadily. The 2004 International Renewable Energy Conference in Bonn put forward the goal of promoting the development of global renewable energy, requiring countries to formulate action plans. In October 2010, in Delhi, India, At the fourth Renewable Energy Conference, the participating countries reached a new consensus [1].

China, which is the world's largest importer of crude oil in 2017, has overtaken Japan as the world's first-largest importer of gas in 2018. China, as the world's largest producer and consumer of energy, faces many problems and challenges, despite its success in energy transformation[2].

\section{The development trend of renewable energy in the world's typical countries}

According to the World Bank's Report on Sustainable Energy Regulation (RISE), issued in 2017, Denmark has a total of 94 points in the sustainable energy policy and management framework score, ranking first in the world[3]. In 2017, the Danish energy consumption fell by
0.4 per cent on a year-on-year basis, reaching 170.3 million tons of oil equivalent, But the consumption of renewable energy increased by $14 \%$, accounting for $27.2 \%$ of the energy consumption. With wind power as an example, Denmark has become the world's largest wind power installation in the total power generation machine, and the wind power has been able to meet the whole country in $201747.9 \%$ of the total electricity demand [4].

Germany ranks first in Europe in installed capacity and electricity generation, is the largest electricity market in Europe, and is also one of the more developed countries in the world. By the end of 2014, Germany's renewable energy has accounted for $30 \%$ of the total power generation scale, the total installed capacity reached $88 \mathrm{GW}$, exceeding the peak load, and the highest instantaneous value accounted for $78 \%$ of Germany's electricity consumption [5]. German experience shows that the development and utilization of renewable energy can meet the electricity demand of industrialized countries on the premise of ensuring the safety and reliability of the power grid.

Since the 1970 s, according to its own energy policy, policy orientation and regulation system, Japan has been exploring and practicing the mode suitable for its energy development. Up to now, its energy efficiency and economic benefits have reached the highest level in the world.

\section{Analysis of the present situation of energy in China.}

According to the statistics of the Ministry of Natural Resources, as of the end of 2017, China's coal has identified a reserve of 170 million tons, an increase of 
4.3 per cent compared with 2016 , an increase of 1.2 per cent for oil, an increase of 1.2 per cent, a natural gas of 550 million cubic metres, an increase of 1.6 per cent, a shale gas of 198.29 billion cubic metres, an increase of 62 per cent, and a coal-bed gas of 3025.4 billion cubic metres, Reduce by $9.5 \%[6]$. Although China's energy resources are rich, the energy development is difficult and the supply capacity is insufficient. According to BP statistics, as of the end of 2017 China's coal storage ratio is 39 , the oil is 18.3 , and the natural gas is 36.7[7]. In addition, countries with strong energy demand in recent years and those with a lack of resources are actively working to promote resource development and procurement through state-owned enterprises, and the fierce competition around resources is on stage and has a major impact on China's energy security.

In recent years, China's photovoltaic, wind and hydropower installed capacity has ranked first in the world, becoming the largest country in the world to save energy and use renewable energy. In 2018, China's renewable energy installed capacity accounted for $29.6 \%$ of the global total, of which hydropower, wind power, solar energy and biomass energy accounted for $27.2 \%$, $32.8 \%, 36 \%$ and $11.4 \%$, respectively. China's renewable energy generation and installed capacity basically achieved the National Renewable Energy Development 13th five-year Plan 2020 target (1.9 trillion kilowatt-hours, 680 million kilowatts).Electricity generation reached 1.87 trillion kilowatt-hours, an increase of about 170 billion kilowatt-hours over the same period last year, and renewable energy generation accounted for 26.7 percent of the total electricity generation, up 0.2 percentage points from the same period last year. Renewable energy power generation reached 728 million kilowatts, an increase of 12 percent over the same period last year, accounting for 38.3 percent of the total installed electricity, up 1.7 percent from the same period last year [8]. However, the existing power grid system is difficult to meet the requirements of large-scale grid connection of renewable energy. Grid constraints lead to the abandonment of wind, water and light in some areas. About 69.1 billion kilowatt-hours of abandoned water capacity in 2018. In 2005, about 27.7 billion kilowatt hours of wind power and 5.49 billion kilowatt hours of abandoned light power were abandoned. In addition, the replacement costs of "replacing coal with gas" and "replacing coal with electricity" are high, the optimal allocation of clean energy is hindered throughout the country, the situation of "heavy construction and light utilization" is more prominent, the supply and demand are unbalanced and uncoordinated, and the proportion of renewable energy in primary energy consumption is still lower than that in advanced countries.

\section{Application of Advanced Technology and equipment}

In order to meet the large-scale renewable energy grid-connected power generation in the construction of distribution network upgrading, German power grid enterprises have advanced technical equipment in the aspects of distribution network structure planning, transferable supply rate enhancement method, line cable planning, intelligent equipment deployment, market-oriented trading mechanism implementation and so on. The research concept and implementation results, draw lessons from foreign advanced technology, combined with the national conditions of our country, improve the utilization of renewable energy technology in our country.

\subsection{Application of Intelligent Voltage Regulation and capacity regulating Transformer in low Voltage Distribution Network Section}

At present, it is still very difficult to feed distributed energy into distribution network, which will continue to increase with the progress of photovoltaic power generation and wind energy expansion plan. according to DINEN50160 standard, in order to maintain the standard voltage range of $\pm 10 \%$, because there are no other control equipment operated by power grid at medium voltage level, So the total tolerance range allocated in the medium voltage and low voltage power grid is $20 \%$. For the distributed energy production, according to the latest FNN criterion, the low voltage grid provides $3 \%$, the medium voltage grid provides $2 \%$, and the remaining $15 \%$ is also used to control the installation in addition to the voltage drop during the power supply period. The system technical tolerance of the installation and actuator is solved effectively by using the intelligent voltage regulating and capacity regulating transformer VRDT in the low voltage distribution network section.

\subsection{Application of distribution network edge voltage control system.}

At present, distributed power generation is developing rapidly, but the infrastructure and equipment technology are relatively backward, so it is difficult to completely solve the fluctuation of renewable energy power generation and the risk of intermittent power generation, which greatly reduces the security and reliability of power grid operation. In addition, with the increase of industrial and residential peak load demand, the heavy load overload of line equipment leads to the increase of line voltage drop, which leads to the decrease of line terminal voltage. Thus, the problem of "low voltage" is caused, which leads to the accelerated damage of lines and equipment and the reduction of the stability of power system.

To solve the above problems, Varentec launched ENGOTM distribution network edge voltage control system, installed in the near end of the load hardware equipment (ENGOV10) to achieve voltage monitoring and reactive volt-ampere regulation, while using the software management platform (ENGOManager) to achieve visual data Real - time monitoring of ENGO V 10. This distributed regulation mode responds faster and adjusts more accurately than the central monitoring mode. 


\section{5 conclusion}

The utilization of energy is directly related to the future development of China's economy. Therefore, the development and utilization of new energy and renewable energy is related to the future development of our country. In the process of previous development, there has been a great consumption of natural ecological energy, which has led to the corresponding environmental pollution in different regions of our country. In order to improve the level of harmonious development between man and nature, it is necessary to develop and utilize renewable energy reasonably, improve the efficiency of energy utilization, play an important role in energy use, gradually realize the virtuous circle of energy use, and improve the comprehensive development level of society.

\section{References}

1. Dong Shuhua,Jiang Jianfei. (2019) Research on the Countermeasures of Renewable Energy Development and Utilization in China. Modern economic information.366.

2. Zhang Suo,Ma Boyong. (2019) the development trend of world energy and the future development direction of energy in China. China's land and resources economy .1-13.

3. $[\mathrm{EB} / \mathrm{OL}] \cdot[2018-08-23]$.Middle Country business Department of Administration.

http://www.mofcom.gov.cn/article/i/jyjl/k/201702/2 0170202523054.shtml.

4. Wang Ning. (2019) the main measures and implications for the development of renewable energy in Denmark. The economy is vertical and horizontal. $111-120$.

5. Hong Lei, Jia Feng, Wu Ke.(2014) Current situation of Renewable Energy Development and Utilization in Germany .Journal of Hefei University (Natural Science Edition), 24 (3): 78-82.

6. Ling Yueming, Ju Jianhua, Jiang Wenbiao, et al. (2018)China Mineral Resources report . Beijing: geological Publishing House.

7. BP. (2018)Yearbook of World Energy Statistics .

8. Guojia energy source bureau. (2019)2018 renewable energy source and network operation situation.

http:/www.nea.gov.cn/2019-01/28/c_137780519.ht $\mathrm{m}$. 